\title{
Abnormal Cardiac Function in the Streptozotocin-Diabetic Rat Changes in Active and Passive Properties of the Left Ventricle
}

Sheldon E. Litwin, Thomas E. Raya, Peter G. Anderson, Sherry Daugherty, and Steven Goldman

Department of Internal Medicine, Veterans Administration Medical Center, Tucson, Arizona 85723; and University Heart Center, University of Arizona College of Medicine, Tucson, Arizona 85724

\begin{abstract}
To provide an integrated assessment of changes in systolic and diastolic function in diabetic rats, we measured conscious hemodynamics and performed ex vivo analysis of left ventricular passive-elastic properties. Rats given streptozotocin (STZ) 65 $\mathrm{mg} / \mathrm{kg}$ i.v. $(n=14)$ were compared with untreated agematched controls $(n=15)$ and rats treated with insulin after administration of STZ $(n=11)$. After $7 \mathrm{~d}$, diabetic rats exhibited decreases in heart rate and peak developed left ventricular (LV) pressure during aortic occlusion. After $26 \mathrm{~d}$ of diabetes there were significant decreases in resting $L V$ systolic pressure, developed pressure, and maximal $+\mathrm{dP} / \mathrm{dt}$, whereas $\mathrm{LV}$ end-diastolic pressure increased and the time constant of $\mathrm{LV}$ relaxation was prolonged. The passive $L V$ pressure-volume relationship was progressively shifted away from the pressure axis, and the overall chamber stiffness constant was decreased. However, "operating chamber stiffness" calculated at end-diastolic pressure was increased at $7 \mathrm{~d}$, and unchanged at $26 \mathrm{~d}$. LV cavity/wall volume and end-diastolic volume were increased after $26 \mathrm{~d}$ of diabetes. Myocardial stiffness was unchanged at both time intervals. All of the above abnormalities were reversed by the administration of insulin. We conclude that the hemodynamic and passive-elastic changes that occur in diabetic rats represent an early dilated cardiomyopathy which is reversible with insulin. (J. Clin. Invest. 1990. 86:481-488.) Key words: diabetes $\bullet$ diastole $\bullet$ passive-elastic $\bullet$ systolic function • ventricular relaxation
\end{abstract}

\section{Introduction}

Diabetes mellitus is associated with the development of myocardial dysfunction in the absence of coronary artery disease, systemic hypertension, or valvular heart disease $(1,2)$. Experimental models of diabetes, especially the streptozotocin (STZ) ${ }^{1}$ rat, have been used to help define the pathophysiology of this disorder $(3,4)$. These studies, which utilized isolated papillary muscle and perfused heart preparations, suggest that

This study was presented in part at the Federation of American Societies for Experimental Biology (FASEB) 1989 scientific session, New Orleans, LA.

Dr. Anderson is with the Department of Pathology, University of Alabama in Birmingham.

Address reprint requests to Dr. Litwin, Cardiology 111C, Tucson VAMC, Tucson, AZ 85723.

Received for publication 29 August 1989 and in revised form 21 February 1990.

1. Abbreviations used in this paper: LV, left ventricular; STZ, streptozotocin.

The Journal of Clinical Investigation, Inc.

Volume 86, August 1990, 481-488 the cardiomyopathy of diabetes mellitus is associated with abnormalities of systolic performance and impairment of left ventricular (LV) relaxation.

The effect of diabetes on LV diastolic function has not been thoroughly examined. The studies which have been done have produced conflicting results. For example, in diabetic dogs the diastolic pressure-volume relation was reported to shift toward the pressure axis, which would indicate decreased chamber compliance, increased myocardial stiffness, or both (5). Conversely, in the STZ rat, the pressure-volume relation of the isolated left ventricle is shifted away from the pressure axis, suggesting increased chamber compliance (4). In neither study was the entire diastolic pressure-volume relation examined, nor was myocardial stiffness measured. Furthermore, the influence of $\mathrm{LV}$ relaxation abnormalities on the shape and position of the diastolic pressure-volume relation were not taken into account. Thus, the previous studies have not adequately resolved the question of whether diabetes is associated with abnormalities of the passive-elastic properties of the heart, independent of changes in relaxation.

Our goal was to characterize the changes in diastolic function (i.e., $\mathrm{LV}$ relaxation and passive-elastic behavior) with diabetes and their relation to alterations in systolic function. We measured conscious hemodynamics, open-chest $L V$ developed pressure, and ex vivo $\mathrm{LV}$ pressure-volume relations. We postulated that the previously described decrease in LV compliance might reflect abnormalities of the contraction-relaxation sequence, rather than changes in intrinsic passive-elastic properties of the myocardium. We also treated a group of diabetic rats with insulin, after a period of untreated diabetes, to establish whether the changes in cardiac function were reversible. This is the first study to integrate hemodynamic data from conscious animals, with a quantitative analysis of the mechanical properties of the heart in diabetes.

\section{Methods}

Diabetes was induced by a single tail vein injection of STZ at a dose of $65 \mathrm{mg} / \mathrm{kg}$ (Sigma Chemical Co., St. Louis, MO) in male SpragueDawley rats $(220-250 \mathrm{~g})$. Diabetic animals were divided into three groups. Group I $(n=5)$ was studied after $7 \mathrm{~d}$ of untreated diabetes. Group II $(n=9)$ was studied after 21-28 d (mean $=26)$ of untreated diabetes. Group III $(n=11)$ was untreated for $21 \mathrm{~d}$, then were treated with subcutaneous NPH human insulin ( $8 \mathrm{U} / \mathrm{kg}$ twice daily) for $21 \mathrm{~d}$. Insulin was not given on the morning of the study. Untreated, agematched rats $(n=15)$ served as the control group. All animals were fed standard rat laboratory diet and were allowed free access to water. Animals were housed two to three per cage and were maintained on a 12-h light-dark cycle.

$L V$ performance in conscious rats. To obtain conscious measurements of LV performance, rats were anesthetized with inhaled methoxyflurane. A 1-mm diam micromanometer-tipped catheter (Millar Instruments, Houston, TX) was passed via the right carotid artery into the LV under constant pressure monitoring. The zero pressure baseline was obtained by placing the pressure sensor in $37^{\circ} \mathrm{C}$ saline before 
insertion. The left femoral vein was cannulated with Silastic tubing which was advanced to the thoracic inferior vena cava. Both catheters were then secured and exteriorized to the dorsal cervical region. Rats were allowed to recover for $4 \mathrm{~h}$. Left ventricular $\mathrm{d} P / \mathrm{d} t$ was obtained from a differentiating circuit in the physiologic recorder (model 2400, Gould, Inc., Cleveland, $\mathrm{OH}$ ) with the high-frequency filter cutoff set at $100 \mathrm{~Hz}$. In conscious rats $100-150$ consecutive beats of $L V$ pressure were recorded directly onto a computer (model AT, IBM Corp., White Plains, NY) and digitized at $1,000 \mathrm{~Hz}(6,7)$. An example of an LV pressure recording in a conscious rat is shown in Fig. 1. These rats were then used for determination of peak developed LV pressure and the LV pressure-volume relationship as described below.

$L V$ relaxation. From the recordings of $L V$ pressures and $d P / d t, L V$ relaxation was determined in untreated diabetic, treated diabetic, and control rats using previously established methods $(6,7)$. Briefly, the decay of LV pressure with time can be closely approximated by the exponential relation:

$P=P_{\mathrm{B}} \mathrm{e}^{-a t}+P_{\mathrm{A}}$

where $P_{\mathrm{B}}+P_{\mathrm{A}}$ is $\mathrm{LV}$ pressure at maximum negative $\mathrm{LV} \mathrm{d} P / \mathrm{d} t, P_{\mathrm{A}}$ is $\mathrm{LV}$ asymptote pressure, and $a$ is the constant of the exponential relation. The value of $\tau$ is calculated by plotting the derivative of pressure with time versus pressure. LV pressures from $100-150$ consecutive beats were analyzed. For each cardiac cycle maximum positive and negative $\mathrm{d} P / \mathrm{d} t$, peak systolic and end-diastolic pressure points were identified. The value of end-diastolic pressure for each cycle was subtracted from each pressure point on the pressure decay curve of that cycle beginning at maximum negative $\mathrm{d} P / \mathrm{d} t$ and ending at a point equal to end-diastolic pressure. The resulting values were then fit to the relation:

$\mathrm{d} P / \mathrm{d} t=-a\left(P-P_{\mathrm{A}}\right)$.

$a$ is calculated by the least squares method, and $\tau=-1 / a$.

Open-chest determinations of peak developed LV pressure. After conscious hemodynamics were recorded, rats were anesthetized with $100 \mathrm{mg} / \mathrm{kg}$ thiobutarbital (Inactin, Buikgulden Pharmaceutical, Konstanz, FRG) i.p., intubated, and ventilated with a volume-cycled respirator (Harvard Apparatus Co., Inc., S. Natick, MA). A left anterior thoracotomy was performed and a snare was placed around the ascending aorta. While recording LV pressure with the previously positioned Millar catheter, an abrupt aortic occlusion was produced by sudden tightening of the snare. Peak developed pressure was defined as the mean of the systolic pressure minus the end-diastolic pressure of the first five stable beats after aortic occlusion.

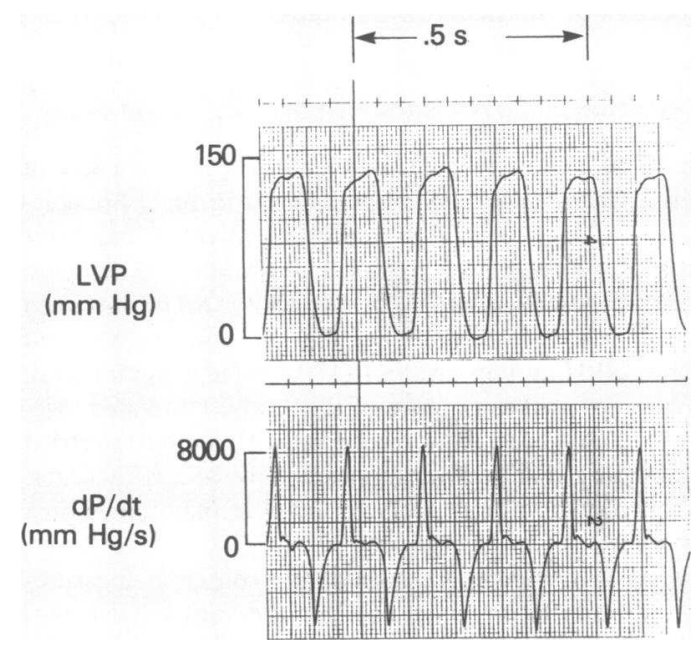

Figure $1 . \mathrm{LV}$ pressure measured in conscious normal rat with solidstate micromanometer-tipped catheter. Peak positive and negative $\mathrm{d} P / \mathrm{d} t$, and $\tau$ are derived from these pressure measurements.
Isolated LV pressure-volume relationship. Pressure-volume data were recorded using methods previously described $(6,7)$. After the measurement of peak developed pressure, potassium chloride ( $2 \mathrm{meq} /$ $\mathrm{ml}$ ) was injected through the femoral vein catheter to arrest the heart. The heart was rapidly removed and the right ventricle was incised. A double lumen catheter, attached to a pressure transducer (Statham 23 Id, Gould, Inc.) and an infusion pump (Sage model 341, Sage Instruments Div., Cambridge, MA) was passed into the left ventricle. The atrioventricular groove was identified and a ligature was passed around the heart and tied to isolate the left atrium from the left ventricle. After gentle aspiration of the $\mathrm{LV}$ cavity to remove any residual blood, and to reduce pressure to $-5 \mathrm{~mm} \mathrm{Hg}$, normal saline was infused at 0.70 $\mathrm{ml} / \mathrm{min}$ into the suspended left ventricle while pressure was recorded. Saline was infused until the pressure increased to $40 \mathrm{~mm} \mathrm{Hg}$. Two or three curves were obtained from each ventricle within $10 \mathrm{~min}$ of cardiac arrest, and before onset of rigor mortis. An example of a pressure-volume recording is shown in Fig. 2.

From the pressure-volume data recorded ex vivo, the overall chamber stiffness constant $K_{c}$ was determined. At least 15 pairs of simultaneous pressure-volume points from each curve were digitized and stored. The pressure-volume data were fitted to the monoexponential equation:

$P=P_{0} \mathrm{e}^{K_{c} V}+P_{\mathrm{B}}$

(mean correlation coefficient $r=0.98 \pm 0.002$ ), where $P$ and $V$ are pressure and volume respectively, and $P_{0}$ is a modeling constant. $P_{\mathrm{B}}$ is defined below. Differentiating this equation with respect to volume yields

$\mathrm{d} P / \mathrm{d} V=K_{\mathrm{c}}\left(P-P_{\mathrm{B}}\right)$.

$\mathrm{d} P / \mathrm{d} V$ is calculated numerically from the continuous pressure recording and plotted vs. pressure. $K_{\mathrm{c}}$ and $P_{\mathrm{B}}$, the pressure intercept, are then calculated by the method of least squares. This derivation allows for the possibility of the curve passing through the zero pressure-volume point. For purposes of comparison, ventricular volumes were indexed to body weight (milliliters per kilogram) and to LV wall volume, because body weight indexing may not be appropriate in animals that lose weight (8). Operating chamber stiffness $\left(C_{\mathrm{s}}\right)$ was defined as

$C_{\mathrm{s}}=K_{\mathrm{c}}\left(P-P_{\mathrm{B}}\right)$,

where $P=$ end-diastolic pressure measured in the conscious animal. This calculation was also done using both forms of indexing.

Myocardial stiffness. The incremental modulus $\left(E_{\mathrm{INC}}\right)$ and the myocardial stiffness constant $\left(K_{\mathrm{m}}\right)$ were calculated assuming a spherical geometry (inner and outer radii $=a$ and $b$, respectively) for the left ventricle, according to previously described methods $(7,9)$. Briefly, midwall radial stress $(\sigma)$ at each pressure point was calculated from the measured values for LV cavity volume and wall volume (mass/1.05) using the expression:

$\sigma=(3 / 2) P\left(V / V_{\mathrm{w}}\right)\left(b^{3} / R^{3}\right)$,

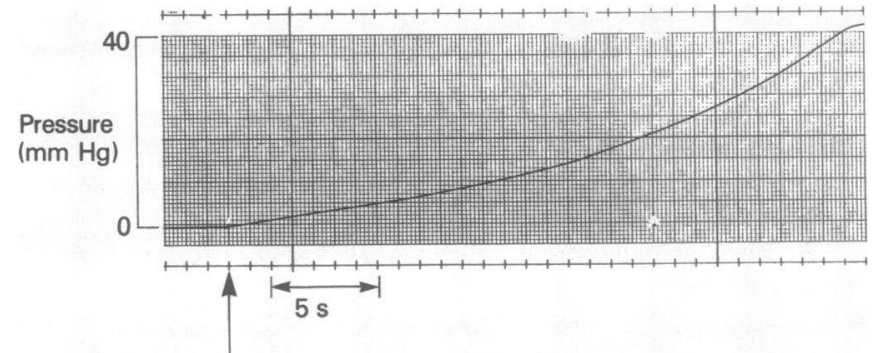

Figure 2. Example of an ex vivo LV pressure-time recording. LV volume is calculated from the time and the known rate of volume inflow. Chamber and myocardial stiffness constants, and LV end-diastolic volume are derived from these curves. 
Table I. Body and Heart Weights and Glucose Levels in Rats with STZ-induced Diabetes

\begin{tabular}{lcccc}
\hline & $\begin{array}{c}\text { Control } \\
(n=15)\end{array}$ & $\begin{array}{c}\text { Group I } \\
(n=5)\end{array}$ & $\begin{array}{c}\text { Group II } \\
(n=9)\end{array}$ & $\begin{array}{c}\text { Group III } \\
(n=11)\end{array}$ \\
\hline Body weight $(\mathrm{kg})$ & $0.28 \pm 0.02$ & $0.25 \pm 0.005$ & $0.23 \pm 0.01$ & $0.35 \pm 0.01$ \\
Left ventricle weight $(g)$ & $0.60 \pm 0.03$ & $0.55 \pm 0.02$ & $0.51 \pm 0.03^{*}$ & $0.67 \pm 0.02$ \\
Left ventricle weight/body weight $(\mathrm{g} / \mathrm{kg})$ & $2.13 \pm 0.05$ & $2.19 \pm 0.06$ & $2.23 \pm 0.04$ & $2.11 \pm 0.04$ \\
Left ventricle/tibia $(\mathrm{g} / \mathrm{cm})$ & $0.188 \pm 0.004$ & & $0.146 \pm 0.006^{\ddagger}$ & $0.196 \pm 0.011$ \\
Glucose $(\mathrm{mmol} / \mathrm{liter})$ & $9.1 \pm 0.6$ & $31.8 \pm 0.7^{\ddagger}$ & $36.2 \pm 2.0^{\ddagger}$ & $21.1 \pm 7.1$
\end{tabular}

Group I, untreated diabetes for $7 \mathrm{~d}$; group II, untreated diabetes for $26 \mathrm{~d}$; group III, insulin treatment for $26 \mathrm{~d}$ after $26 \mathrm{~d}$ of untreated diabetes.

* $P<0.05$ compared with control; ${ }^{\ddagger} P<0.01$ compared with control.

where

$R=(a+b) / 2$,

$V=4 / 3 \pi a^{3}$,

and

$V_{\mathrm{w}}=4 / 3 \pi b^{3}-4 / 3 \pi a^{3}$

The incremental modulus is obtained from the formula:

$E_{\mathrm{INC}}=1 / 2 \Delta \sigma(\Delta R / R)$

The relation between the incremental modulus and stress is linear and can be described by the equation:

$E_{\mathrm{INC}}=K_{\mathrm{m}} \sigma+c$

where $K_{\mathrm{m}}$, the slope of the line, is the myocardial stiffness constant, and $c$ is a constant.

Cavity/wall volume. Ventricular cavity volume at a distending pressure of $10 \mathrm{~mm} \mathrm{Hg}$ was determined from the passive pressure-volume relation. After completion of the pressure-volume recordings, the heart was separated into right ventricle, and left ventricle plus septum, and weighed. $\mathrm{LV}$ wall volume $\left(V_{\mathrm{w}}\right)$ was determined from the mass of the left ventricle, such that $V_{w}=L V$ mass/1.05 (density of muscle). Operating end-diastolic volume was calculated from the pressure-volume curves using the measured end-diastolic pressure from the conscious animal.

$L V$ weight/tibia length. Because body weight may be an unreliable reference for normalizing heart weight in situations where body weight changes, LV weight was normalized to both body weight and tibia length to determine whether hypertrophy was present. Tibias were prepared using previously described methods $(8)$.

Glucose levels. Blood was drawn just prior to $\mathrm{KCl}$ arrest at the conclusion of each study. It was immediately spun, and the serum was frozen at $-20^{\circ} \mathrm{C}$ for later analysis. Glucose was measured in the thawed serum using a glucose oxidase method (model CX3 glucose analyzer, Beckman Instruments, Inc., Fullerton, CA).

Histology. Hearts were immersion fixed in $10 \%$ neutral buffered formalin. The heart was serially sliced into $3-\mathrm{mm}$ sections perpendicular to the axis of the heart from apex to base. These sections were embedded in parafin, sectioned at $5 \mu \mathrm{m}$, and serial sections were stained with hematoxylin-eosin and with Gomori's aldehyde fuchsin trichrome stain to highlight collagen.

Statistical analysis. Differences between the three diabetic and control groups were detected using analysis of variance. Where differences were found, significance was determined with Dunnett's test for multiple comparisons with a single control (10). Values for LV chamber stiffness constants and $\tau$ were determined by regression analysis using the method of least squares. Results are presented as mean \pm SEM. Statistical significance was defined at the $P<0.05$ level.

\section{Results}

Diabetes was confirmed by the finding of glycosuria $2 \mathrm{~d}$ after the injection. None of the rats showed more than trace ketonuria. Severe hyperglycemia was seen in the untreated diabetic rats (Table I). Diabetic animals had lower body weights and left ventricle weights, but left ventricle/body weight was unchanged compared to control (Table I). Left ventricle/tibia length was decreased in group II animals.

Conscious hemodynamics and peak developed pressure. Heart rate was progressively slowed, and systolic function was impaired in the diabetic rats (Table II). LV systolic pressure, and maximal positive $\mathrm{d} P / \mathrm{d} t$ were unchanged at $7 \mathrm{~d}$ (group I), but decreased at 4 wk (group II) of untreated diabetes. Peak developed pressure in open-chest rats was decreased in group I, and further decreased in group II. LV end-diastolic pressure had a tendency to increase in group I, and was significantly increased in group II.

Indices of diastolic function. Relaxation of the left ventricle in conscious rats, as assessed by the time constant of isovolumic pressure decay $(T)$, and maximal negative $\mathrm{d} P / \mathrm{d} t$, was slowed in group II (Table III). There were also alterations in the passive-elastic properties of the ventricle. The overall chamber stiffness constant $\left(K_{\mathrm{c}}\right)$ was decreased in group II based on LV wall volume indexing, but not body weight indexing. The ex vivo pressure-volume relations with LV volume indexed to body weight are shown in Fig. 3. In contrast to overall chamber stiffness, operating chamber stiffness $\left(C_{\mathrm{s}}\right)$ was increased in group I, and unaltered in group II using both forms of indexing. Fig. 4 shows the pressure-volume relations of the control and untreated diabetic rats with the tangent lines at mean end-diastolic pressure. The slope of the tangent $=C_{\mathrm{s}}$. Operating chamber stiffness is influenced by the end-diastolic pressure, and hence may be different than the overall chamber stiffness constant. The myocardial stiffness constant $\left(K_{\mathrm{m}}\right)$ was unchanged in diabetic rats (Table III). The stiffness-stress relations are linear (Fig. 5). There are no significant differences in the slope or position of the lines. Ventricular dilatation developed in groups I and II, as defined by increases in end-diastolic volume. This was accompanied by an increase in cavity/wall volume $\left(V / V_{\mathrm{w}}\right)$ in group II (Table III).

Effects of insulin therapy. Administration of insulin resulted in weight gain and lowering of serum glucose (Table I). All conscious hemodynamic variables, and peak developed LV pressure returned to baseline after 3-4 wk of treatment with insulin (Table II). Treatment was associated with the correc- 
Table II. Conscious Hemodynamic Data and Peak Developed LV Pressure in Control and STZ-induced Diabetic Rats

\begin{tabular}{|c|c|c|c|c|}
\hline & $\begin{array}{l}\text { Control } \\
(n=15)\end{array}$ & $\begin{array}{l}\text { Group I } \\
(n=5)\end{array}$ & $\begin{array}{l}\text { Group II } \\
(n=9)\end{array}$ & $\begin{array}{l}\text { Group III } \\
(n=11)\end{array}$ \\
\hline Heart rate (beats/min) & $372 \pm 5$ & $329 \pm 12^{\ddagger}$ & $307 \pm 11^{\ddagger}$ & $407 \pm 6$ \\
\hline LV systolic pressure $(\mathrm{mm} \mathrm{Hg})$ & $150 \pm 2$ & $146 \pm 13$ & $124 \pm 5^{\ddagger}$ & $144 \pm 4$ \\
\hline $\mathrm{LV}$ end-diastolic pressure $(\mathrm{mm} \mathrm{Hg})$ & $8 \pm 2$ & $15 \pm 2$ & $15 \pm 3^{*}$ & $7 \pm 1$ \\
\hline $\mathrm{d} P / \mathrm{d} t(m m H g / s)$ & $9,609 \pm 171$ & $9,948 \pm 1,229$ & $7,015 \pm 344^{\ddagger}$ & $9,699 \pm 865$ \\
\hline$-\mathrm{d} P / \mathrm{d} t(m m H g / s)$ & $8,253 \pm 257$ & $6,952 \pm 929$ & $5,193 \pm 339^{\ddagger}$ & $7,324 \pm 390$ \\
\hline Peak developed $\mathrm{LV}$ pressure $(\mathrm{mm} \mathrm{Hg})$ & $217 \pm 9$ & $182 \pm 10^{*}$ & $133 \pm 3^{\ddagger}$ & $217 \pm 9$ \\
\hline
\end{tabular}

Group I, untreated diabetes for $7 \mathrm{~d}$; group II, untreated diabetes for $26 \mathrm{~d}$; group III, insulin treatment for $26 \mathrm{~d}$ after $26 \mathrm{~d}$ of untreated diabetes. ${ }^{*} P<0.05$ compared with control, ${ }^{\ddagger} P<0.01$ compared with control.

tion of the abnormalities of diastolic function, including LV relaxation, LV end-diastolic pressure and volume, and LV chamber stiffness (Table III).

Histology. Qualitative light microscopy revealed no histologic changes in myocardium from diabetic animals. Specifically, there was no evidence of interstitial collagen accumulation seen with stain specific for collagen (Fig. 6).

\section{Discussion}

The major findings of this study were that both active and passive properties of the left ventricle were altered in conscious diabetic rats. The former was manifest as impairment of $\mathrm{LV}$ relaxation and systolic function, while the latter consisted of decreased LV chamber stiffness, unchanged myocardial stiffness, and increased LV end-diastolic volume. Despite a decrease in the overall chamber stiffness constant, operating chamber stiffness was increased at 1 wk and unchanged at 4 wk. This seemingly paradoxical change in operating chamber stiffness resulted from the opposing effects of increased end-di-

Table III. Indices of Diastolic Function in Control and STZ-induced Diabetic Rats

\begin{tabular}{lrccc}
\hline & $\begin{array}{c}\text { Control } \\
(n=15)\end{array}$ & $\begin{array}{c}\text { Group I } \\
(n=5)\end{array}$ & $\begin{array}{c}\text { Group II } \\
(n=9)\end{array}$ & $\begin{array}{c}\text { Group III } \\
(n=11)\end{array}$ \\
\hline$T(m s)$ & $13.1 \pm 0.3$ & $15.1 \pm 1.0$ & $16.5 \pm 0.6^{\ddagger}$ & $12.5 \pm 1.0$ \\
$K_{\mathrm{c}}\left(V_{\mathrm{w}}\right)$ & $4.4 \pm 0.4$ & $4.4 \pm 0.6$ & $3.0 \pm 0.2^{*}$ & $4.9 \pm 0.3$ \\
$K_{\mathrm{c}}(\mathrm{BW})$ & $2.0 \pm 0.2$ & $2.1 \pm 0.3$ & $1.5 \pm 0.1$ & $2.5 \pm 0.2$ \\
$C_{\mathrm{s}}\left(V_{\mathrm{w}}\right)$ & $27.9 \pm 6.6$ & $68.3 \pm 15.2^{*}$ & $46.0 \pm 9.6$ & $42.4 \pm 6.3$ \\
$C_{\mathrm{s}}(\mathrm{BW})$ & $12.6 \pm 2.7$ & $32.8 \pm 7.2^{*}$ & $22.3 \pm 4.7$ & $21.0 \pm 8.75$ \\
$K_{\mathrm{m}}$ & $7.3 \pm 0.1$ & $7.3 \pm 0.4$ & $7.3 \pm 0.3$ & $8.1 \pm 0.3$ \\
$\mathrm{EDV}(\mathrm{BW})$ & & & & \\
$\quad(m l / k g)$ & $0.72 \pm 0.11$ & $1.54 \pm 0.28^{\ddagger}$ & $1.74 \pm 0.15^{\ddagger}$ & $0.83 \pm 0.1$ \\
$V / V_{\mathrm{w}}$ & $0.54 \pm 0.04$ & $0.67 \pm 0.09$ & $0.74 \pm 0.03^{*}$ & $0.51 \pm 0.05$ \\
& & & & \\
\hline
\end{tabular}

Group I, untreated diabetes for $7 \mathrm{~d}$; group II, untreated diabetes for $26 \mathrm{~d}$; group III, insulin treatment for $26 \mathrm{~d}$ after $26 \mathrm{~d}$ of untreated diabetes. Abbreviations: EDV, LV end-diastolic volume ( $\mathrm{ml} / \mathrm{kg}) ; \mathrm{BW}$, indexed to body weight; $C_{\mathrm{s}}$, operating chamber stiffness; $K_{\mathrm{c}}$, chamber stiffness constant; $K_{\mathrm{m}}$, myocardial stiffness constant; $T$, time constant of left ventricular relaxation; $V / V_{\mathrm{w}}, \mathrm{LV}$ cavity/wall volume at $10 \mathrm{~mm} \mathrm{Hg}$ distending pressure; $V_{\mathrm{w}}$, indexed to $\mathrm{LV}$ wall volume. ${ }^{*} P<0.05$ compared with control; ${ }^{*} P<0.01$ compared with control. astolic pressure, and decreased chamber stiffness. All of the hemodynamic and mechanical abnormalities were corrected by insulin therapy. No histologic alterations in the myocardium of diabetic rats were evident on light microscopy. Thus, diabetic rats have characteristics of an early dilated cardiomyopathy which is due to metabolic, rather than structural, alterations of the myocardium.

Cardiac performance is a function of ventricular filling and ejection. As such, it is important to understand the variables which effect both systole and diastole when studying a given disease state. We have attempted to dissect systole and diastole into components which could be quantitatively analyzed. Measurements of active processes, i.e., relaxation and developed pressure, were done in intact rats. The passive-elastic properties of the myocardium, i.e., overall chamber stiffness $\left(K_{\mathrm{c}}\right)$, operating chamber stiffness $\left(C_{\mathrm{s}}\right)$, and myocardial stiffness $\left(K_{\mathrm{m}}\right)$, were evaluated ex vivo. This type of analysis of LV function provides a framework to understand cardiovascular function in diabetes and may help to explain conflicting data from previous studies.

Effects of diabetes on systolic function and ventricular relaxation. In conscious rats, resting systolic function, assessed by $\mathrm{LV}$ systolic pressure and $\mathrm{d} P / \mathrm{d} t$, was normal after $7 \mathrm{~d}$ of

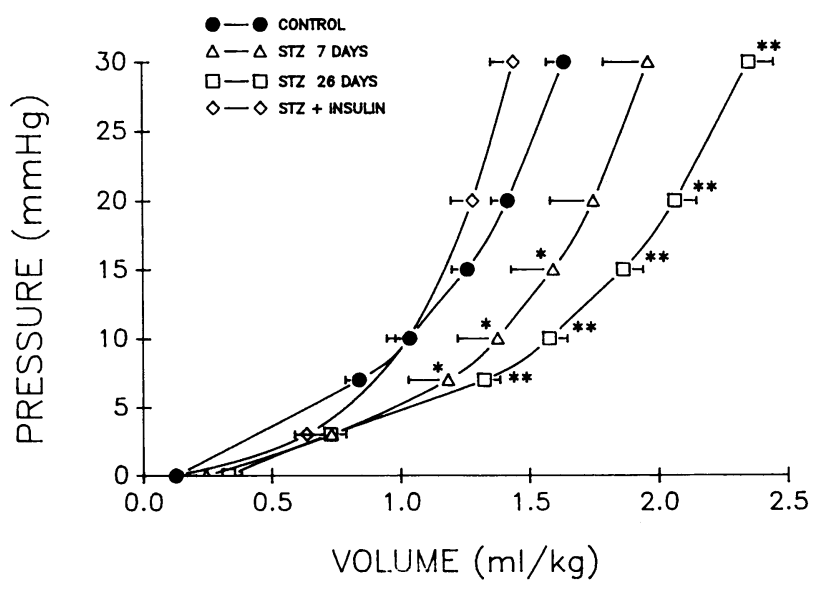

Figure 3. Ex vivo left ventricular pressure-volume relations. Note that in diabetic rats the curves are progressively shifted away from the pressure axis. Insulin treatment restores the normal pressure-volume relation. Error bars show SEM for volume at each pressure. ${ }^{*} P$ $<0.05 ;{ }^{* *} P<0.01$ compared with control. 


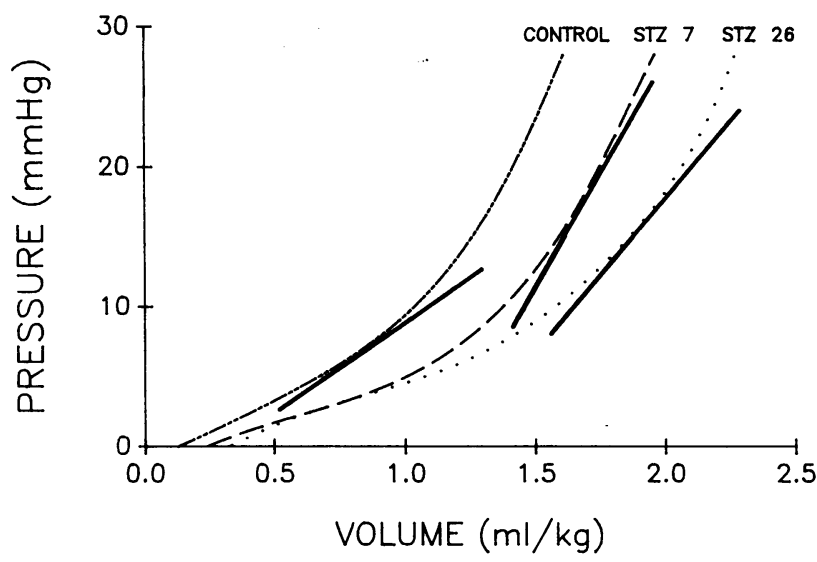

Figure 4. Operating chamber stiffness in control and untreated diabetic rats. The curves shown are the best monoexponential fitted to the data points. The slope of the pressure-volume curves (tangent line) at mean end-diastolic pressure for each group = operating chamber stiffness. This illustrates how operating chamber stiffness can be increased (group I), and unchanged (group II) when the overall chamber stiffness constants are unchanged (group I), and decreased (group II).

untreated diabetes. However, systolic reserve was compromised, as evidenced by decreased peak developed LV pressure in open-chest rats. After 3-4 wk of diabetes, both resting and stressed systolic function were abnormal. These observations in conscious and anesthetized rats support the findings in studies of papillary muscle from diabetic rats, in which time to peak tension was increased and peak rate of tension rise was decreased (3). Likewise, in previous studies using isolated perfused hearts from diabetic rats, peak LV pressure response to progressive increases in preload was attenuated (4).

One of the best measures of relaxation in vivo is the time constant of ventricular pressure decay $(\tau)$. We found that $\tau$ was progressively prolonged in conscious diabetic rats. Ventricular relaxation is altered by changes in temperature (11), catecholamines (12), inotropic state (13), heart rate (14), and loading conditions (15). Diabetes is associated with several of these factors, including impaired contractility, bradycardia, increased blood volume (16), and increased catecholamines (17). Since the combined effect of these factors is difficult to predict,

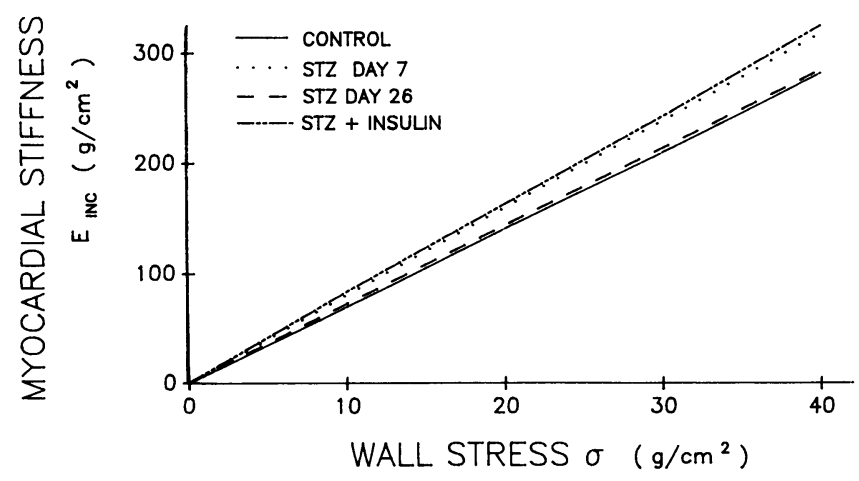

Figure 5. Diastolic stiffness-stress relations of the left ventricle in diabetic and control hearts. The lines represent the best linear fit for the stiffness-stress points for the rats in each group (mean correlation coefficient $r=0.98$ ). The curves are not significantly different. it is important to document the rate of ventricular relaxation in conscious rats. The prolongation of $\tau$ that we observed expands on the findings of previous in vitro studies, which showed prolongation of time to one-half relaxation and depressed peak rate of tension fall in papillary muscles, and decreased $-\mathrm{d} P / \mathrm{d} t$ in isolated hearts $(3,4)$.

The mechanisms of impaired systolic function and slowed relaxation are unclear. These changes may share a common mechanism since both are related to the binding or release of calcium from troponin C. It is likely that altered sarcoplasmic reticular calcium transport plays a role in these changes (18, 19). Large increases in heart rate $(100 \%)$ have been shown to decrease $\tau$ by $\sim 20 \%$ in conscious dogs (14). Thus, part of the slowed rate of relaxation seen in the diabetic rats may be attributable to bradycardia; however, it is unlikely that bradycardia alone was responsible for slowed relaxation since heart rate was only decreased by $17 \%$ in the diabetic rats, while $\tau$ was increased by $26 \%$. Bradycardia and loss of normal baroreflexes have been previously observed in diabetic rats $(16,20)$. These findings may reflect decreased sensitivity to adrenergic stimulation, or increased sensitivity to muscarinic stimulation (21, 22). Alterations in adrenergic tone may have contributed to slowed relaxation in the diabetic rats.

Effects of diabetes on passive-elastic properties of the ventricle. Diastolic filling is determined by a complex interaction of ventricular relaxation, passive-elastic properties of the ventricle, myocardial blood flow, atrial pressure and contractility, and ventricular interaction (23). Early diastolic filling is influenced predominantly by the rate of ventricular relaxation, mid-diastolic filling is determined more by the mechanical and geometric characteristics of the ventricle (chamber stiffness), and late diastolic filling depends on the force of atrial contraction. It is difficult to separate the varying effects of these forces. In our study, the passive-elastic properties of the left ventricle were studied in the arrested heart prior to rigor mortis; thus, the above confounding variables were all eliminated and we were able to study passive filling over the full range of physiologic pressures.

The passive pressure-volume relationship is curvilinear and is uniquely determined for a given ventricle by myocardial stiffness, and the ratio of ventricular cavity and wall volume $\left(V / V_{\mathrm{w}}\right)(9)$. An increase in end-diastolic pressure will increase "operating chamber stiffness" by causing a shift to a steeper portion of the curve. Pure volume expansion, or a decline in systolic function alone can cause this type of change (23). This movement along the curve should be distinguished from an actual change in position, or shape of the entire curve, which represents a primary change in diastolic properties (23). In the present study, myocardial stiffness was unchanged, and both $V$ and $V / V_{\mathrm{w}}$ were increased. Thus, passive chamber stiffness $\left(K_{\mathrm{c}}\right)$ was decreased. The changes in passive stiffness should not be viewed in isolation. There are concurrent changes in systolic and diastolic function in this model. The sum of these changes results in a pressure-volume curve that is less steep and shifted away from the pressure axis. However, LV end-diastolic pressure rises before LV dilatation occurs, and the result is an increase in operating chamber stiffness at $7 \mathrm{~d}$. After $26 \mathrm{~d}$ operating chamber stiffness returns to baseline due to the opposing effects of the increased end-diastolic pressure, which causes a shift to a steeper portion of the curve, and the decrease in the slope of the pressure-volume curve. 

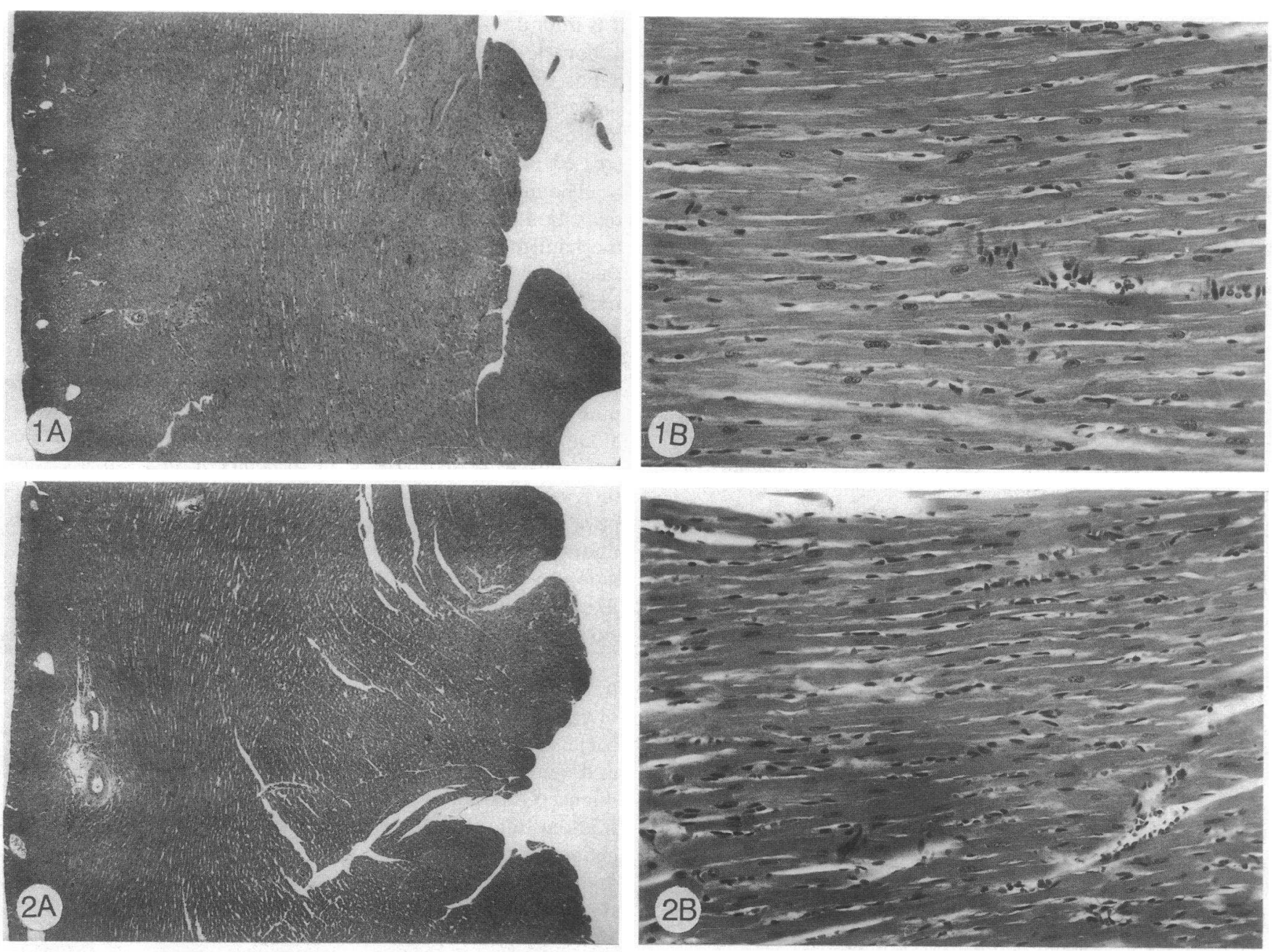

Figure 6. Light microscopy from representative control and diabetic hearts. Transmural low power $(\times 30)$ photomicrographs of control $(1 A)$ and diabetic $(2 A)$ free wall show no significant lesions in diabetic heart. Higher power magnification $(\times 450)$ of control $(1 B)$ and $(\times 425)$ diabetic $(2 B)$ heart tissue demonstrates normal myocyte morphology in the subendomyocardial region of the left ventricle.

The forces which govern ventricular dilatation are not well understood. In the diabetic rat chronic systolic dysfunction almost certainly contributes to the LV dilatation. This may not be the same in humans or other animal models of diabetes. Prolonged increases in end-systolic volume are believed to cause ventricular remodelling due to slippage of myocytes (24). Diabetes is also thought to be associated with a hyperadrenergic state (17). It is possible that this sympathetic stimulation results in arterial and venoconstriction which could alter loading conditions, and thus play a role in the ventricular dilatation seen in this model. Blood volume has been reported to increase in experimental diabetes, which also could predispose to ventricular dilatation (16). A final factor which could contribute to $\mathrm{LV}$ dilatation is increased ventricular filling time due to bradycardia.

Effects of insulin treatment. All of the hemodynamic abnormalities were reversed with insulin therapy. This confirms similar findings from previous studies in rats, but differs from observations in diabetic dogs $(16,25,26)$. It cannot be ascertained from currently available data whether it is the hyperglycemia itself, the lack of insulin, or some combination of the two which causes the cardiomyopathy of diabetes. The finding that insulin markedly increases calcium uptake in response to catecholamine stimulation of the diabetic heart supports the contention that insulin is an important determinant of cardiac function (27). Furthermore, treatment with insulin causes normalization of myosin isoforms, which shift to a fetal pattern in the diabetic state (28). Alternatively, there is reason to believe that hyperglycemia may directly contribute to the development of the cardiomyopathy. The glycation of intracellular proteins may alter enzyme or receptor function in a manner which depresses cardiac function (29). In support of such a mechanism are the findings of increased protein kinase $\mathrm{C}$ activity and decreased Na-K ATPase activity in retinal capillary endothelial cells cultured in high-glucose medium (30). Similarly, increased protein kinase $\mathrm{C}$ activity and decreased polyphosphoinositol turnover have been observed in glomeruli from diabetic rats (31).

Relationship of the present study to previous work. Our findings of decreased overall chamber stiffness with normal myocardial stiffness are at variance with previous investigations in which an exaggerated increase in LV end-diastolic pressure during intraventricular saline infusion, was interpreted as increased myocardial stiffness (5). The latter study 
was done in a chronic ( $1 \mathrm{yr}$ ) canine model of mild alloxan-induced diabetes. There are several potential explanations for the difference between the studies. First, it is possible that the effects of diabetes on the heart are species-specific. Histologic characteristics of the myocardium appear to be different in various experimental models of diabetes. Chronically diabetic dogs have been reported to have increased periodic acid-Schiff staining of interstitial glycoproteinlike material in the ventricular wall (5) and increased collagen content (26), whereas our findings strengthen previous observations that diabetic rats do not have histologic evidence of fibrosis (32) or increased collagen content (33). Lack of significant alterations in myocardial histology support the concept that the functional changes we observed have a metabolic, rather than a structural basis.

Secondly, the effects of diabetes may be time-dependent. If so, chamber and myocardial stiffness in the rat might increase after a longer duration of hyperglycemia. The deposition of interstitial advanced glycation products occurs later in the course of diabetes and potentially could contribute to alterations in passive myocardial stiffness (29). It is not presently known whether this process occurs in the heart. Furthermore, in other models of cardiomyopathy, such as the spontaneously hypertensive rat, increases in myocardial stiffness occur only after a prolonged period of hypertension (34).

Finally, methodologic differences may be important. For example, in the previously cited study: (a) pressure-volume data were gathered over a relatively small pressure range; $(b)$ only two pressure-volume points were recorded for each animal; (c) no attempt was made to fit the data to an exponential curve, nor to quantify chamber stiffness constants; $(d)$ possible contributions of baroreflexes were not taken into account; and (e) measurements were made at end-diastole and thus may reflect viscous effects related to atrial contraction, rather than true passive behavior. The severity of the diabetes may also affect the findings of a given study.

We believe that some of the changes in systolic and diastolic function that we have described in the rat may be common to other animal species, and to patients with diabetes as well. The exaggerated end-diastolic pressure response to intraventricular saline infusion in previous experiments (5) could be explained by impaired relaxation, which is known to shift the pressure-volume curve upwards. Large-volume loads, such as those used in that study, have been associated with prolongation of relaxation (15). To separate the effects of relaxation abnormalities from changes in myocardial stiffness in intact animals, we measured the passive properties of the myocardium in potassium arrested, ex vivo hearts. In this preparation, the heart is arrested with potassium chloride, and persistent cross-bridge activation should not complicate the analysis. This technique allowed us to determine that changes in passive muscle stiffness were not responsible for the increased end-diastolic pressure and volume.

Clinical significance. In most cardiac diseases diastolic abnormalities are thought to be a consequence of systolic dysfunction, although symptoms referable to isolated diastolic dysfunction may predominate in certain patients. Recent data suggest that diastolic dysfunction may contribute to the increased mortality rate seen in diabetic patients after myocardial infarction (35). Further evidence of diastolic dysfunction in patients with diabetes comes from Doppler echocardiographic evaluation of trans-mitral diastolic flow in diabetic patients without ischemic heart disease (36). Diabetic patients were observed to have a decrease in the early/late (E/A) flow velocity, which is suggestive of impaired relaxation. At present, there is still considerable debate about the use of noninvasive indices of diastolic function and the meaning of isolated abnormalities in a given index (37). This lack of consensus makes study of diastolic function in humans difficult. Although there are significant differences between STZ-rats and humans with diabetes, our data are consistent with the speculation that impaired relaxation and contractility may contribute to the cardiac dysfunction seen in diabetic patients. Moreover, our findings emphasize that the early cardiovascular dysfunction of diabetes is reversible with insulin replacement.

\section{Acknowledgments}

We gratefully acknowledge the technical assistance of Chuck Hougland for writing the computer programs used in this study, and the laboratory work provided by Teresa Westhoff and Michelle Timmons.

This study was supported in part by grants from the Arizona Affiliate of the American Heart Association, Arizona Disease Control Research Commission, the Glaxo Corporation, the National Institutes of Health (HL-20984), and the Veterans Administration.

\section{References}

1. Kannel, W. B., M. Hjortland, and W. P. Castelli. 1974. Role of diabetes in congestive heart failure: the Framingham study. Diabetes. 34:29-34.

2. Regan, T. J., M. M. Lyons, S. S. Ahmed, G. E. Levinson, H. A. Oldewurtel, M. R. Ahmad, and B. Haider. 1977. Evidence for cardiomyopathy in familial diabetes mellitus. J. Clin. Invest. 60:885-899.

3. Fein, F. S., L. B. Kornstein, J. E. Strobeck, J. M. Capasso, and E. H. Sonnenblick. 1980. Altered myocardial mechanics in diabetic rats. Circ. Res. 47:922-933.

4. Penpargkul, S., T. Schaibel, T. Yipintsoi, and J. Scheuer. 1980. The effect of diabetes on performance and metabolism of rat hearts. Circ. Res. 47:911-921.

5. Regan, T. J., P. O. Ettinger, M. I. Khan, M. U. Jesrani MU, M. M. Lyons, H. A. Oldewurtel, and M. Weber. 1974. Altered myocardial function and metabolism in chronic diabetes mellitus without ischemia in dogs. Circ. Res. 35:222-237.

6. Raya, T. E., R. G. Gay, L. Lancaster, M. Aguirre, C. Moffett, and S. Goldman. 1988. Serial changes in left ventricular relaxation and chamber stiffness after large myocardial infarction in rats. Circulation. 77:1424-1431.

7. Gay, R. G., T. E. Raya, L. D. Lancaster, R. W. Lee, E. Morkin, and S. Goldman. 1988. Effects of thyroid state on venous compliance and left ventricular performance in rats. Am. J. Physiol. 254:H81-88.

8. Yin, F. C. P., H. A. Spurgeon, K. Rakusan, M. L. Weisfeldt, and E. G. Lakatta. 1982. Use of tibial length to quantify cardiac hypertrophy: application in the aging rat. Am. J. Physiol. 243:H941-947.

9. Mirsky, I. 1984. Assessment of diastolic function: suggested methods and future considerations. Circulation. 69:836-839.

10. Dunnett, C. W. 1964. New tables for multiple comparisons with a control. Biometrics. 20:482-491.

11. Courtois, M. R., P. B. Kurnik, and P. A. Ludbrook. 1988. Sensitivity of isovolumic relaxation to hypothermia during myocardial infarction. J. Am. Coll. Cardiol. 11:201-206.

12. Weiss, J. L., J. W. Fredericksen, and M. L. Weisfeldt. 1976. Hemodynamic determinants of the time-course of fall in canine left ventricular pressure. J. Clin. Invest. 58:751-760.

13. Parmley, W. W., and E. H. Sonnenblick. 1969. Relation between mechanics of contraction and relaxation in mammalian cardiac muscle. Am. J. Physiol. 216:1084-1091.

14. Freeman, G. L., W. C. Little, and R. A. O'Rourke. 1987. 
Influence of heart rate on left ventricular performance in conscious dogs. Circ. Res. 61:455-464.

15. Raff, G. L., and S. A. Glantz. 1981. Volume loading slows left ventricular isovolumic relaxation rate: evidence of load-dependent relaxation in the intact dog heart. Circ. Res. 48:813-824.

16. Carbonell, L. F., M. G. Salom, J. Garcia-Estan, F. J. Salazar, M. Ubeda, and T. Quesada. 1987. Hemodynamic alterations in chronically conscious unrestrained diabetic rats. Am. J. Physiol. 252:H900905.

17. Christensen, N. J. 1974. Plasma norepinephrine and epinephrine in untreated diabetics, during fasting and after insulin administration. Diabetes. 23:1-8.

18. Ganguly, P. K., G. N. Pierce, K. S. Dhalla, and N. S. Dhalla. 1983. Defective sarcoplasmic reticular transport in diabetic cardiomyopathy. Am. J. Physiol. 244:E528-535.

19. Penpargkul, S., F. Fein, E. H. Sonnenblick, and J. Scheuer. 1981. Depressed sarcoplasmic reticular function for diabetic rats. $J$. Mol. Cell Cardiol. 13:303-309.

20. Chang, K. S. K., and D. D. Lund. 1986. Alterations in the baroreceptor reflex control of heart rate in streptozotocin diabetic rats. J. Mol. Cell. Cardiol. 18:617-624.

21. Atkins, F. L., R. T. Dowell, and S. Love. 1985. B-adrenergic receptors, adenylate cyclase activity, and cardiac dysfunction in the diabetic rat. J. Cardiovasc. Pharmacol. 7:66-70.

22. Aronstam, R. S., and G. O. Carrier. 1989. Insulin prevention of altered muscarinic receptor-G protein coupling in diabetic rat atria. Diabetes. 38:1611-1616.

23. Gilbert, J. C., and S. A. Glantz. 1989. Determinants of left ventricular filling and of the diastolic pressure-volume relation. Circ. Res. 64:827-852.

24. Weisman, H. F., D. E. Bush, F. J. A. Mannisi, M. L. Weisfeldt, and B. Healy. 1988. Cellular mechanisms of myocardial infarct expansion. Circulation. 78:186-201.

25. Fein, F. S., J. E. Strobeck, A. Malhotra, J. Scheuer, and E. H. Sonnenblick. 1981. Reversibility of diabetic cardiomyopathy with insulin in rats. Circ. Res. 49:1251-1261.

26. Regan, T. J., C. F. Wu, K. Y. Chien, H. A. Oldewurtel, and B.
Haider. 1981. Myocardial composition and function in diabetes: the effects of chronic insulin use. Circ. Res. 49:1268-1277.

27. Gotzsche, O. 1985. Abnormal myocardial calcium uptake in streptozotocin-diabetic rats: evidence for a direct insulin effect on catecholamine sensitivity. Diabetes. 34:287-290.

28. Dillman, W. H. 1980. Diabetes mellitus induces changes in cardiac myosin of the rat. Diabetes. 29:579-582.

29. Brownlee, M., H. Vlassara, and A. Cerami. 1984. Nonenzymatic glycosylation and the pathogenesis of diabetic complications. Ann. Intern. Med. 101:527-537.

30. Lee, T. S., L. C. MacGregor, S. J. Fluharty, and G. L. King. 1989. Differential regulation of protein kinase $\mathrm{C}$ and $(\mathrm{Na}, \mathrm{K})$-adenosine triphosphatase activities by elevated glucose levels in retinal capillary endothelial cells. J. Clin. Invest. 83:90-94.

31. Craven, P. A., and F. R. DeRubertis. 1989. Protein kinase C activation in glomeruli from streptozotocin diabetic rats: possible mediation by glucose. J. Clin. Invest. 83:1667-1675.

32. Gotsche, O. 1982. Lack of cardiotoxic effect of isoproterenol in streptozotocin diabetic rats: a morphometric study of isoproterenol induced fibrosis. Virchows Arch. Pathol. Anat. 397:83-91.

33. Modrak, J. 1980. Collagen metabolism in the myocardium from streptozotocin-diabetic rats. Diabetes. 29:547-550.

34. Mirsky, I., J. M. Pfeffer, M. A. Pfeffer, and E. Braunwald. 1983. The contractile state as the major determinant in the evolution of left ventricular dysfunction in the spontaneously hypertensive rat. Circ. Res. 53:767-778.

35. Stone, P. H., J. E. Muller, T. Hartwell, B. J. York, J. D. Rutherford, C. B. Parker, Z. G. Turi, H. W. Strauss, J. T. Willerson, T. Robertson, et al. 1989. The effect of diabetes mellitus on prognosis and serial left ventricular function after acute myocardial infarction: contribution of both coronary artery disease and diastolic left ventricular dysfunction to the adverse prognosis. J. Am. Coll. Cardiol. 14:49-57.

36. Zarich, S. W., B. E. Arbuckle, L. R. Cohen, M. Roberts, and R. W. Nesto. 1988. Diastolic abnormalities in young asymptomatic diabetic patients assessed by pulsed Doppler echocardiography. J. Am. Coll. Cardiol. 12:114-120.

37. Lew, Y. W. Y. 1989. Evaluation of left ventricular diastolic function. Circulation. 79:1393-1397. 